\title{
Two Triads of Squares
}

\author{
By J. Lagrange and J. Leech
}

\begin{abstract}
The thirteen points $(0,0),\left( \pm a_{i}, 0\right), i=1,2,3,\left(0, \pm b_{j}\right), j=1,2,3$, will be at integer distances from one another if the two triads $a_{1}^{2}, a_{2}^{2}, a_{3}^{2}, b_{1}^{2}, b_{2}^{2}, b_{3}^{2}$ are such that the nine sums $a_{i}^{2}+b_{j}^{2}$ are all perfect squares. Infinite families of solutions are derived from solutions of $\{m, n\}^{2}=\{p, q\}\{r, s\}$, where $\{m, n\}=\left(m^{2}-n^{2}\right) / 2 m n$, etc. Additional numerical examples are given. Two solutions are given in which one of the triads is extended to a tetrad.
\end{abstract}

1. Introduction. Several investigations have been made of sets of points in a plane such that all the distances between pairs of them are rational numbers. (For references, see [1, Problem D 20]; for a recent survey, see [2].) The rational points on a line form an infinite set, and it is also easy to find infinite sets on a circle (for example, points with coordinates $(\cos 4 \theta, \sin 4 \theta)$, where $\tan \theta$ ranges over rational numbers). Our interest therefore lies in sets of points which do not all lie on one line or circle. In particular, we may seek sets of points which maximize the number $N$ such that, whatever line or circle we choose, there are at least $N$ of the points of the set not on the chosen line or circle. For example, Leech [5] constructs sets of nine points of which no line or circle contains more than four, so $N=5$ for these sets. In this paper, we construct sets of thirteen points of which no line contains more than seven and no circle more than four, so $N=6$ for these sets.

The present sets of points comprise the point of intersection of two perpendicular lines, which we take as axes of coordinates, together with points $\left( \pm a_{i}, 0\right), i=1,2,3$, and $\left(0, \pm b_{j}\right), j=1,2,3$, for integers $a_{i}, b_{j}$. All their distances will be integers if the two triads of squares $a_{1}^{2}, a_{2}^{2}, a_{3}^{2}$ and $b_{1}^{2}, b_{2}^{2}, b_{3}^{2}$ are such that the nine sums $a_{i}^{2}+b_{j}^{2}$ are all perfect squares. These are the triads of the title. To each such pair of triads there corresponds a reciprocal pair, obtained by replacing each $a_{i}$ and $b_{j}$ by the quotient on dividing it into the LCM of all the $a_{i}$ and $b_{j}$.

To avoid frequent writing of fractions, we use the following notation. Let $\{m, n\}$ designate the fraction $\left(m^{2}-n^{2}\right) / 2 m n$, and let $\{m, n\}^{\prime}$ designate its reciprocal $2 m n /\left(m^{2}-n^{2}\right)$. (It will always be assumed that $m n\left(m^{2}-n^{2}\right) \neq 0$.) Then $\{m, n\}\{m, n\}^{\prime}=1$, and $\{m, n\}^{\prime}=\{m+n, m-n\}$. In numerical examples, we remove any common factors to ensure that $m, n$ are coprime, and we use the latter relation to ensure that they are of opposite parity.

2. An Infinity of Rational Squares $a_{i}^{2}+b_{1}^{2}, a_{i}^{2}+b_{2}^{2}$. It is straightforward to construct infinite sets of points at rational distances of which four are of the form $\left(0, \pm b_{1}\right),\left(0, \pm b_{2}\right)$, and the others are $(0,0)$ and $\left( \pm a_{i}, 0\right)$ for $i=1,2, \ldots$, with all the $a_{i}$ rational [6]. To find one such point $\left(a_{1}, 0\right)$ with $a_{1} \neq 0$, we have to make $a_{1}^{2}+b_{1}^{2}$

Received May 23, 1984; revised May 28, 1985.

1980 Mathematics Subject Classification (1985 Revision). Primary 11D09. 
and $a_{1}^{2}+b_{2}^{2}$ squares simultaneously. This can be achieved by setting $b_{1} / a_{1}=\{p, q\}$ and $a_{1} / b_{2}=\{r, s\}$, so we require $b_{1} / b_{2}=\{p, q\}\{r, s\}$. Writing $x / z=p / q$ and $y / z=(r+s) /(r-s)$, we express this as

$$
b_{1} x\left(y^{2}-z^{2}\right)=b_{2} y\left(x^{2}-z^{2}\right),
$$

which is a plane cubic curve in the homogeneous coordinates $x, y, z$. It has eight rational points of finite order, namely $(1,0,0),(0,1,0),(0,0,1),\left(b_{1}, b_{2}, 0\right)$ and the four unit points $( \pm 1, \pm 1,1)$, which form a closed set. In the special case that $b_{1} / b_{2}=\{m, n\}^{2}$, there are eight further rational points of finite order, namely $x / z= \pm m / n$ or $\pm n / m$, each with $y / z=(x+z) /(x-z)$ or $(z-x) /(z+x)$. Otherwise, there are no further rational points of finite order. So if we have any ratio $b_{1} / b_{2}$ which is expressible in the form $b_{1} / b_{2}=\{p, q\}\{r, s\}$ with $\{r, s\} \neq\{p, q\}$, then this corresponds to rational points of infinite order on the cubic curve (1), and there are an infinity of points $\left( \pm a_{i}, 0\right)$ at rational distances from $\left(0, \pm b_{1}\right)$ and $\left(0, \pm b_{2}\right)$ (and of course from each other). For these infinite sets, we thus have $N=4$, which seems to be the largest value known for infinite sets.

3. Solutions of $\{m, n\}^{2}=\{p, q\}\{r, s\}$. In the special case $b_{1} / b_{2}=\{m, n\}^{2}$ mentioned above, there may be additional representations $b_{1} / b_{2}=\{p, q\}\{r, s\}$, corresponding to rational points of infinite order on the cubic curve. We develop these in some detail as we shall use them in Section 5 to solve our main problem of constructing pairs of triads of squares. An infinite family of such ratios are found by solving simultaneously the Diophantine equations $\xi^{2}+\eta^{2}=\zeta^{2}$ and $\zeta^{2}-\xi \eta=\rho^{2}$. For any such solution, we have $b_{1} / b_{2}=\xi^{2} / \eta^{2}=\{\eta, \rho\}\{\rho, \xi\}^{\prime}$. It is another elliptic curve problem to solve these equations; solutions are generated by $\xi / \eta=\{4,1\}$, $-\{52,17\},\{3247,1560\},-\{571663,436440\}, \ldots$. Another infinite family of solutions are found by solving simultaneously the Diophantine equations $\xi^{2}+\eta^{2}=\zeta^{2}$ and $\eta^{2}+\xi \zeta=\rho^{2}$. For any such solution, we have $b_{1} / b_{2}=\xi^{2} / \eta^{2}=\{\xi+\zeta, \rho\}\{\rho, \xi\}^{\prime}$. It is another elliptic curve problem to solve these equations; solutions are generated by $\xi / \eta=\{4,1\}^{\prime},\{17,4\},-\{76,15\}^{\prime},-\{570,353\},\{4785,2584\}^{\prime},\{83777,43384\}, \ldots$ A third infinite family of solutions are found by solving simultaneously the Diophantine equations $\xi^{2}+\eta^{2}=\zeta^{2}$ and $\eta \zeta+\zeta \xi+\xi \eta=\rho^{2}$. For any such solution, we have $b_{1} / b_{2}=\xi^{2} / \eta^{2}=\{\xi+\zeta, \rho\}\{\eta+\zeta, \rho\}^{\prime}$. It is yet another elliptic curve problem to solve these equations; solutions are generated by $\xi / \eta=-\{8,5\},\{541,184\}$, $-\{399401,78384\}, \ldots$. Some additional solutions of $\{m, n\}^{2}=\{p, q\}\{r, s\}$, not of these special forms, have been found by a short computer search, described in Section 4.

As solutions correspond to points of infinite order on the cubic curve (1), from any one solution we can construct an infinity of others with the same values of $m$ and $n$; these involve integers of rapidly increasing size. Solutions, however, come in pairs involving integers of similar size. If a point of infinite order on the curve is joined to one of the second set of eight points of finite order, such as $x / z=m / n$, $y / z=(x+z) /(x-z)=(m+n) /(m-n)$, then the third point of intersection of this line with the curve corresponds to the other of this pair of solutions. The solution paired with $\{m, n\}^{2}=\{p, q\}\{r, s\}$ is

$$
\begin{aligned}
\{m, n\}^{2}= & \{(m p+n q)(m s+n r),(m q+n p)(m r+n s)\} \\
& \times\{(m p+n q)(m r-n s),(m q+n p)(m s-n r)\} .
\end{aligned}
$$


4. Numerical Examples of $\{m, n\}^{2}=\{p, q\}\{r, s\}$. The following pairs of solutions were found by a computer search for solutions $\{m, n\}^{2}=\{p, q\}\{r, s\}^{\prime}$ having $n<m \leqslant 20$ and either $q<p \leqslant 500$ or $s<r \leqslant 500$; we include a pair for $\{15,4\}^{2}$ found in an unsystematic extension of this search.

$$
\begin{aligned}
&\{4,1\}^{2}=\{13,8\}\{14,1\}=\{19,8\}\{22,3\} \\
&\{5,2\}^{2}=\{61,54\}^{\prime}\{183,160\}=\{323,106\}\{682,325\} \\
&\{5,4\}^{2}=\{315,296\}\{494,235\}=\{527,274\}\{2200,2047\} \\
&\{8,5\}^{2}=\{50,23\}\{96,73\}=\{380,341\}\{515,112\} \\
&=\{159,26\}^{\prime}\{512,265\}=\{163,24\}\{2363,2200\} \\
&=\{235,194\}\{451,160\}=\{304,101\}\{1044,875\} \\
&\{9,2\}^{2}=\{424,395\}^{\prime}\{5760,4187\}=\{1349,422\}\{4738,729\} \\
&\{9,8\}^{2}=\{65,48\}\{113,108\}=\{67,66\}\{513,224\} \\
&\{13,6\}^{2}=\{96,79\}\{722,553\}^{\prime}=\{143,86\}\{702,229\} \\
&\{14,3\}^{2}=\{136,129\}^{\prime}\{1161,896\}=\{294,47\}\{403,114\} \\
&\{15,4\}^{2}=\{596,273\}\{1700,237\}=\{722,177\}^{\prime}\{3456,295\} \\
&\{15,8\}^{2}=\{66,43\}\{800,327\}=\{108,95\}\{464,65\} \\
&\{17,4\}^{2}=\{34,15\}\{152,17\}=\{296,23\}\{578,319\}
\end{aligned}
$$

The simplest numerical solutions of the first two infinite families given in Section 3 form the first pair; that of the third family appears as $\{8,5\}^{2}=\{50,23\}\{96,73\}$. These solutions were given in $\left[3\right.$, p. 88], together with the solution $\{52,17\}^{2}=$ $\{3637,1768\}\{3026,611\}$, the second solution of the first family. The second solution of the second family appears above; it is $\{17,4\}^{2}=\{296,23\}\{578,319\}$.

On the cubic curve for $\{8,5\}^{2}$, the three pairs of solutions correspond to independent points of infinite order. We have found no other example with more than one independent point of infinite order in this range of search. However, the third solution of the second family gives the pair

$$
\{76,15\}^{2}=\{3930,209\}\{4139,2280\}=\{675,248\}\{722,71\},
$$

and we find the further independent pair

$$
\{76,15\}^{2}=\{384,125\}\{7442,5915\}^{\prime}=\{9103,380\}\{23980,14877\} .
$$

Dependent solutions involve proportionately much larger integers. For example, from the simplest pair of solutions for $\{4,1\}^{2}$, we deduce the next pair

$$
\{4,1\}^{2}=\{2204,825\}\{10244,1631\}=\{4976,915\}^{\prime}\{18971,1024\} \text {. }
$$

In any solution of $\{m, n\}^{2}=\{p, q\}\{r, s\}$, the ratios $\{p, q\},\{m, n\},\{r, s\}$ form a geometric progression. Let $u / v$ be its common ratio. Then, the two ratios $u / v$ in a pair of solutions are related by $\left\{u_{1}, v_{1}\right\}\left\{u_{2}, v_{2}\right\}=\left(\xi^{2}+\eta^{2}\right) / 2 \xi \eta$, where $\xi / \eta=$ $\{m, n\}$ (so $\xi^{2}+\eta^{2}=\zeta^{2}$ ), by a straightforward but tedious calculation. This last equation (without the requirement that $\xi^{2}+\eta^{2}$ be square) is the characteristic equation for a cuboid which has its three edges, two of its face diagonals and its 
body diagonal integers ([4, Eq. (4.3)], [1, Problem D 18, Eq. (M)]). Indeed, the simplest solution $\{26,7\}\{190,99\}=\left(15^{2}+8^{2}\right) / 2.15 .8$ appears in [3] (in Table, p. 93, see Note 3 to Table), and in [4, Fig. 1] and [1, Problem D 18, Fig. 11]. This corresponds to the geometric progressions $\{13,8\},\{4,1\},\{14,1\}$ and $\{19,8\},\{4,1\}$, $\{22,3\}$ in the simplest pair above.

The solutions paired with those of the three infinite families of Section 3 do not have correspondingly simple expressions for their generators. However, for the second family, with $\eta^{2}+\xi \zeta=\rho^{2}$, the solution paired with $\xi^{2} / \eta^{2}=\{\xi+\zeta, \rho\}\{\rho, \xi\}^{\prime}$ has smaller generators, and the ratio $u / v$ has the simple expression

$$
u / v=\left(\xi+\frac{1}{2}(\zeta+\rho)\right) /\left(\xi+\frac{1}{2}(\zeta-\rho)\right) .
$$

The simplest examples are $\{4,1\}^{2}=\{13,8\}\{14,1\}$ with $u / v=26 / 7$, and $\{17,4\}^{2}$ $=\{34,15\}\{152,17\}$ with $u / v=585 / 266$. These are used in Section 5 below.

5. Two Triads with $a_{1} / a_{2}=b_{1} / b_{2}$. We now use solutions of $\{m, n\}^{2}=$ $\{p, q\}\{r, s\}$ to construct triads $a_{i}^{2}, b_{j}^{2}$ such that $a_{i}^{2}+b_{j}^{2}$ are squares for all nine pairs $a_{i}, b_{j}$. For any $a_{1}, a_{2}, b_{1}, b_{2}$ with $a_{i}^{2}+b_{j}^{2}$ squares, we can find an infinity of further rational $a_{i}$ such that $a_{i}^{2}+b_{1}^{2}, a_{i}^{2}+b_{2}^{2}$ are squares, and an infinity of further rational $b_{j}$ such that $a_{1}^{2}+b_{j}^{2}, a_{2}^{2}+b_{j}^{2}$ are squares. In general, we shall not have $a_{i}^{2}+b_{j}^{2}$ square for any $i, j>2$, but a certain special assumption enables us to make $a_{3}^{2}+b_{3}^{2}$ square also. Suppose $a_{1}, a_{2}, b_{1}, b_{2}$ satisfy $a_{1} / a_{2}=b_{1} / b_{2}$. We write $a_{1}=u x, a_{2}=v x, b_{1}=u y, b_{2}=v y$; then we require $x^{2}+y^{2},(u x)^{2}+(v y)^{2},(v x)^{2}$ $+(u y)^{2}$ to be squares. Setting $y / x=\{m, n\}, v y / u x=\{p, q\}, u y / v x=\{r, s\}$, we satisfy these requirements with solutions of $\{m, n\}^{2}=\{p, q\}\{r, s\}$, which have been developed in Sections 3 and 4. Suppose now that $b_{3}=w x$, so that to make $a_{1}^{2}+b_{3}^{2}, a_{2}^{2}+b_{3}^{2}$ squares we have to make $u^{2}+w^{2}, v^{2}+w^{2}$ squares. We know that $u / v=\{p, q\}^{\prime}\{m, n\}=\{m, n\}^{\prime}\{r, s\}$, corresponding to points of infinite order on the cubic curve, so there are an infinity of values $b_{3}=w x$, distinct from $b_{1}$ and $b_{2}$, making $a_{1}^{2}+b_{3}^{2}, a_{2}^{2}+b_{3}^{2}$ squares. To each such value there corresponds a value $a_{3}=w y=b_{3} b_{1} / a_{1}=b_{3} b_{2} / a_{2}$ making $a_{3}^{2}+b_{1}^{2}, a_{3}^{2}+b_{2}^{2}, a_{3}^{2}+b_{3}^{2}$ squares simultaneously, since $a_{3} / b_{1}=w / u, a_{3} / b_{2}=w / v$ and $a_{3} / b_{3}=y / x$. We thus have triads of squares $a_{i}^{2}, b_{j}^{2}$ with all nine sums $a_{i}^{2}+b_{j}^{2}$ squares, as required. The reciprocal pair of triads corresponds to the same values of $a_{1}, a_{2}, b_{1}, b_{2}$ but with a new value of $w$ related to the old by $w_{1} w_{2}=u v$. (Rescaling may be needed to ensure integer triads.)

Two solutions of general form are given by

$$
\begin{aligned}
u / v= & \{p, q\}^{\prime}\{m, n\}=\{m, n\}^{\prime}\{r, s\} \\
= & \{(m p+n q)(m s+n r),(m q+n p)(m s-n r)\}^{\prime} \\
& \times\{(m p+n q)(m r-n s),(m p-n q)(m s-n r)\} \\
= & \{(m p+n q)(m r-n s),(m q+n p)(m r+n s)\}^{\prime} \\
& \times\{(m p-n q)(m r+n s),(m p+n q)(m s+n r)\} .
\end{aligned}
$$

In the simplest numerical examples, these give

$$
\begin{aligned}
26 / 7 & =\{13,8\}^{\prime}\{4,1\}=\{4,1\}^{\prime}\{14,1\} \\
& =\{12,5\}^{\prime}\{15,2\}=\{220,171\}^{\prime}\{209,90\}
\end{aligned}
$$


and

$$
\begin{aligned}
190 / 99 & =\{19,8\}^{\prime}\{4,1\}=\{4,1\}^{\prime}\{22,3\} \\
& =\{28,5\}^{\prime}\{21,2\}=\{20,13\}^{\prime}\{13,6\},
\end{aligned}
$$

and lead to the following reciprocal pairs of solutions to our original problem:

$\begin{array}{rrrrrr}952 & 3536 & 1800, & 1785 & 6630 & 960, \\ 23400 & 6300 & 12376, & 12480 & 3360 & 23205 ; \\ 153272 & 569296 & 1128600, & 287385 & 1067430 & 601920, \\ 14671800 & 3950100 & 1992536, & 7824960 & 2106720 & 3736005 ; \\ 5544 & 10640 & 23400, & 10395 & 19950 & 12480, \\ 148200 & 77220 & 35112, & 79040 & 41184 & 65835 ; \\ 18216 & 34960 & 12600, & 34155 & 65550 & 6720, \\ 79800 & 41580 & 115368, & 42560 & 22176 & 216315 .\end{array}$

The first solution above is the smallest of any we have found. Some further solutions of this type, not given by these general expressions, have been found by a short computer search. This examined ratios $u / v=\{p, q\}^{\prime}\{m, n\}=\{m, n\}^{\prime}\{r, s\}$, found as above, for further representations $u / v=\{c, d\}\{e, f\}$ in small integers. One of these is of unusual simplicity. From $\{17,4\}^{2}=\{34,15\}\{152,17\}$ we find $u / v=585 / 266=\{34,15\}^{\prime}\{17,4\}=\{17,4\}^{\prime}\{152,17\}=\{4,1\}\{13,6\}^{\prime}$, giving $u=$ $585, v=266, w=312$, and the triads

\section{$15970572618 \quad 42432,795603617685176$.}

This is the simplest such solution we have found for which $y / x \neq 15 / 8$ (instead, we have $u / w=15 / 8)$.

6. Two Triads, General Case. The foregoing solutions are based on the special assumption $a_{1} / a_{2}=b_{1} / b_{2}$. It is natural to enquire whether there are solutions not of this special form. Here we have no general theory or formulae, but numerical examples have been found by computer searches which indicate that they are not infrequent. The following search method was adopted. Since we require $a_{2} / a_{1}=$ $\left(a_{2} / b_{j}\right)\left(b_{j} / a_{1}\right)$ for $j=1,2,3$, we look for ratios $a_{2} / a_{1}$ which have several representations as products $a_{2} / a_{1}=\{p, q\}\{r, s\}$ in comparatively small integers. A favorable example is

$$
\begin{aligned}
25 / 91 & =\{3,2\}\{10,3\}^{\prime}=\{4,3\}^{\prime}\{13,12\}=\{8,5\}^{\prime}\{8,7\} \\
& =\{11,4\}\{44,5\}^{\prime}=\{14,9\}\{18,5\}^{\prime}=\{19,2\}^{\prime}\{38,13\},
\end{aligned}
$$

which we use to illustrate the method. We choose one of these representations; in this example, $25 / 91=\{8,5\}^{\prime}\{8,7\}$ is successful. Combining this representation with the others, we find several ratios $h / k$ which have representations of the forms $h / k=\{8,5\}\{p, q\}=\{8,7\}\{r, s\}$. Among these are $5 / 128=\{8,5\}\{13,12\}=$ $\{8,7\}\{4,3\}$ and $765 / 1216=\{8,5\}\{38,13\}=\{8,7\}\{19,2\}$. A further search shows that these admit the further representations $5 / 128=\{11,6\}\{17,16\}$ and $765 / 1216$ $=\{11,6\}\{19,8\}$, where we notice the appearance of $\{11,6\}$ in both expressions. This last repetition indicates a solution to our problem: we may take $a_{i} / b_{j}$ to be the $(i, j)$ entry in the array

$$
\left[\begin{array}{rrr}
\{8,7\}^{\prime} & \{4,3\} & \{19,2\} \\
\{8,5\}^{\prime} & \{13,12\} & \{38,13\} \\
\{11,6\}^{\prime} & \{17,16\} & \{19,8\}
\end{array}\right]
$$


whose rows (and likewise columns) are scalar multiples of one another. By transposing the array, or interchanging each entry $\{p, q\}$ with its reciprocal $\{p, q\}^{\prime}$, we obtain the reciprocal solution. In this example the two pairs of triads are

$\begin{array}{rrrrrr}74256 & 20400 & 15444, & 9945 & 254592 & 15808, \\ 376200 & 1369368 & 1808800, & 2808960 & 109725 & 1767150 .\end{array}$

Further arrays, leading to solutions in greater integers, are not infrequent. One which leads to a solution in smaller integers is

$$
\left[\begin{array}{rrr}
\{8,3\}^{\prime} & \{21,4\}^{\prime} & \{35,16\}^{\prime} \\
\{8,7\} & \{17,16\} & \{68,57\} \\
\{28,11\} & \{8,5\} & \{19,6\}
\end{array}\right]
$$

giving the solution

$$
26880412533150,308006800023256,
$$

which is the smallest we have found of this general form.

The search was made for ratios $a_{2} / a_{1}$ with $a_{2}<a_{1} \leqslant 100$ having several representations $a_{2} / a_{1}=\{p, q\}\{r, s\}$ in small integers, and was then extended to other ratios, such as $5 / 128$ and $765 / 1216$, which appeared in the course of the working, as above, to have several such representations. Arrays giving triads appear frequently, but not in order of magnitude. For example, our smallest general solution above does not involve two rows or columns having a ratio $a_{i} / a_{j}$ with $a_{i}<a_{j} \leqslant 100$, and we cannot claim to have exhausted the range up to this smallest solution found.

7. Two Triads with $a_{1} a_{2}=b_{1} b_{2}$. Solutions of a special form appear rather frequently in this search. In these, the array has a minor of the form

$$
\left[\begin{array}{cc}
\{p, q\} & \{r, s\} \\
\{r, s\}^{\prime} & \{p, q\}^{\prime}
\end{array}\right]
$$

corresponding to a pair of triads which satisfy $a_{1} a_{2}=b_{1} b_{2}$. These are, however, more readily found by means of a modified search process, which we illustrate with an example. We choose a ratio with two convenient expressions of the form $\{p, q\}\{r, s\}$; in this example, $25 / 91=\{3,2\}\{10,3\}^{\prime}=\{8,5\}^{\prime}\{8,7\}$ is successful. From these expressions we obtain two associated ratios, here $9 / 28=\{3,2\}^{\prime}\{8,7\}=$ $\{10,3\}^{\prime}\{8,5\}$ and $13 / 64=\{3,2\}\{8,5\}=\{10,3\}\{8,7\}$. We examine other representations of these three ratios, and we find among them $25 / 91=\{11,4\}\{44,5\}^{\prime}$ and $9 / 28=\{11,4\}^{\prime}\{16,11\}$. These lead to the array

$$
\left[\begin{array}{rrr}
\{8,7\} & \{10,3\} & \{3,2\} \\
\{10,3\}^{\prime} & \{8,7\}^{\prime} & \{8,5\}^{\prime} \\
\{16,11\} & \{44,5\} & \{11,4\}
\end{array}\right]
$$

and to the pairs of triads

$$
\begin{array}{rrrrrr}
30030 & 147840 & 85995, & 224224 & 19800 & 72072, \\
20160 & 4095 & 7040, & 2700 & 30576 & 8400 .
\end{array}
$$

In solutions of this form, the relations between the $\{p, q\}$ involved are more symmetrical than is apparent from the foregoing arrangement. We may see this by 
writing out the expressions

$$
\begin{array}{clll}
91 / 25 & =\{3,2\}^{\prime}\{10,3\} & =\{8,5\}\{8,7\}^{\prime} & =\{44,5\}\{11,4\}^{\prime}, \\
9 / 28 & =\{8,5\}\{10,3\}^{\prime} & =\{3,2\}^{\prime}\{8,7\} & =\{16,11\}\{11,4\}^{\prime}, \\
819 / 1408 & =\{16,11\}\{10,3\} & =\{44,5\}\{8,7\} & =\{8,5\}\{11,4\}, \\
63 / 22 & =\{44,5\}\{10,3\}^{\prime} & =\{16,11\}\{8,7\}^{\prime} & =\{3,2\}^{\prime}\{11,4\} .
\end{array}
$$

Each partition of these four rows into two pairs gives rise to an array of the required form and a reciprocal pair of solutions. In this example the two further arrays are

$$
\left[\begin{array}{rrr}
\{11,4\} & \{8,7\}^{\prime} & \{8,5\}^{\prime} \\
\{8.7\} & \{11,4\}^{\prime} & \{44,5\}^{\prime} \\
\{16,11\} & \{3,2\}^{\prime} & \{10,3\}^{\prime}
\end{array}\right] \text { and }\left[\begin{array}{rrr}
\{10,3\}^{\prime} & \{11,4\}^{\prime} & \{44,5\}^{\prime} \\
\{11,4\} & \{10,3\} & \{3,2\} \\
\{16,11\} & \{8,5\} & \{8,7\}
\end{array}\right] \text {, }
$$

and the corresponding pairs of solutions are

$\begin{array}{rrrrrr}9856 & 1575 & 5733, & 11760 & 1320 & 3780, \\ 61425 & 384384 & 105600, & 51480 & 458640 & 160160 ; \\ 18900 & 24024 & 6600, & 28665 & 15840 & 49280, \\ 32032 & 25200 & 91728, & 21120 & 38220 & 12285 .\end{array}$

The first of these is the smallest solution of this form that we have found, though again our search has not been exhaustive.

Another set of relations of this form, giving three arrays and six solutions, is

$\begin{array}{rlll}70 / 117 & =\{28,11\}^{\prime}\{11,6\} & =\{13,12\}\{8,7\}^{\prime} & =\{4,3\}\{8,5\}^{\prime}, \\ 55 / 442 & =\{13,12\}\{11,6\}^{\prime} & =\{28,11\}^{\prime}\{8,7\} & =\{17,16\}\{8,5\}^{\prime}, \\ 5 / 128 & =\{17,16\}\{11,6\} & =\{4,3\}\{8,7\} & =\{13,12\}\{8,5\}, \\ 77 / 170 & =\{4,3\}\{11,6\}^{\prime} & =\{17,16\}\{8,7\}^{\prime} & =\{28,11\}^{\prime}\{8,5\} .\end{array}$

The smallest solution obtained from this set is

$$
663082521120,10296616013600 .
$$

Although the same expressions for 5/128 appear in this set and in the first array of Section 6 , the solutions do not appear to be otherwise related. Other sets that we have found lead only to solutions in still greater integers.

Our first solutions, obtained in Section 5 from solutions of $\{m, n\}^{2}=$ $\{p, q\}\{r, s\}$, can now be seen to be doubly special. The corresponding array has two minors of the form

$$
\left[\begin{array}{cc}
\{m, n\} & \{c, d\} \\
\{c, d\}^{\prime} & \{m, n\}^{\prime}
\end{array}\right]
$$

and one of the form

$$
\left[\begin{array}{rr}
\{m, n\} & \{r, s\} \\
\{p, q\} & \{m, n\}
\end{array}\right],
$$

so it has the form

$$
\left[\begin{array}{ccc}
\{m, n\} & \{c, d\} & \{r, s\} \\
\{c, d\}^{\prime} & \{m, n\}^{\prime} & \{e, f\}^{\prime} \\
\{p, q\} & \{e, f\} & \{m, n\}
\end{array}\right]
$$


For the simplest numerical solution given in Section 5 (with the triads reordered), the array is

$$
\left[\begin{array}{ccc}
\{4,1\} & \{15,2\} & \{14,1\} \\
\{15,2\}^{\prime} & \{4,1\}^{\prime} & \{12,5\}^{\prime} \\
\{13,8\} & \{12,5\} & \{4,1\}
\end{array}\right] .
$$

Our searches have shown that this is the smallest solution of this doubly special form. It is probably the smallest of all solutions, but our searches for solutions of more general form have not been exhaustive, and we cannot be sure of this.

8. A Triad and a Tetrad. Many pairs of otherwise unrelated arrays are found to share a common $2 \times 2$ minor (for example, those involving the ratio 5/128 mentioned above). The frequency of these invites investigation whether pairs of arrays exist sharing a $3 \times 2$ minor. Any such pair would combine into a $3 \times 4$ array and would lead to one of the triads being extended to a tetrad. Our searches have found one such example. The simple ratio $9 / 5$ has the representations $9 / 5=\{2,1\}\{3,2\}^{\prime}$ $=\{20,13\}^{\prime}\{52,25\}$, from which we find the associated ratio $693 / 2080$ which has numerous representations including $693 / 2080=\{2,1\}\{20,13\}=\{3,2\}\{52,25\}=$ $\{28,5\}\{26,23\}=\{36,13\}\{46,35\}$. The last three of these lead to the array

$$
\left[\begin{array}{rrrr}
\{3,2\} & \{52,25\}^{\prime} & \{40,17\}^{\prime} & \{69,56\} \\
\{28,5\} & \{26,23\}^{\prime} & \{51,44\}^{\prime} & \{49,16\} \\
\{36,13\} & \{46,35\}^{\prime} & \{68,49\}^{\prime} & \{16,9\}
\end{array}\right]
$$

and to the triads plus tetrads

$$
\begin{array}{rrrr}
9282000 & 60386040 & 26822600, & \\
22276800 & 7422030 & 8947575 & 44142336
\end{array}
$$

and

$$
\begin{array}{rrrr}
101754576 & 15640800 & 35212320, & \\
42397740 & 127254400 & 105557760 & 21396375 .
\end{array}
$$

We cannot comment on the frequency of such arrays or on the prospects for finding even larger arrays from $3 \times 5$ or $4 \times 4$ upwards. Any example of a $4 \times 4$ array would lead to sets of seventeen points at integer distances with $N=8$.

\footnotetext{
Département de Mathématiques

Université de Reims

B. P. 347

51062 Reims Cedex, France

Department of Computing Science

University of Stirling

Stirling FK9 4LA, Scotland
}

1. R. K. GuY, Unsolved Problems in Number Theory, Springer-Verlag, Berlin and New York, 1981.

2. J. Lagrange, "Points du plan dont les distances mutuelles sont rationelles," Seminaire de Théorie des Nombres de Bordeaux, 1982-1983, Exposé no. 27, 10 pp.

3. J. LEECH, "The location of four squares in an arithmetic progression, with some applications," Computers in Number Theory (A. O. L. Atkin and B. J. Birch, eds.), Academic Press, London and New York, 1971, pp. 83-98.

4. J. LEECH, “The rational cuboid revisited," Amer. Math. Monthly, v. 84, 1977, pp. 518-533.

5. J. LeECH, "Two Diophantine birds with one stone," Bull. London Math. Soc., v. 13, 1981, pp. $561-563$

6. W. D. Peeples, "Elliptic curves and rational distance sets," Proc. Amer. Math. Soc., v. 5, 1954, pp. 29-33. 